\title{
FIXED POINT THEOREMS IN GENERATING SPACES OF QUASI-NORM FAMILY AND APPLICATIONS
}

\author{
JIAN-ZHONG XIAO AND XING-HUA ZHU
}

Received 11 April 2005; Accepted 21 November 2005

Some new concepts of generating spaces of quasi-norm family are introduced and their linear topological structures are studied. These spaces are not necessarily locally convex. By virtue of some properties in these spaces, several Schauder-type fixed point theorems are proved, which include the corresponding theorems in locally convex spaces as their special cases. As applications, some new fixed point theorems in Menger probabilistic normed spaces and fuzzy normed spaces are obtained.

Copyright (c 2006 J.-Z. Xiao and X.-H. Zhu. This is an open access article distributed under the Creative Commons Attribution License, which permits unrestricted use, distribution, and reproduction in any medium, provided the original work is properly cited.

\section{Introduction}

The Schauder fixed point theorem and its generalizations which were obtained by Krasnoselskii et al. (see $[3,5]$, we call them the Schauder-type fixed point theorems), play important role in nonlinear analysis. In classical case, many interesting extensions and important applications of these theorems were presented by Fan [1] and others. In nonclassical case, several extensions of these theorems in Menger probabilistic normed spaces were given under some conditions by Zhang-Guo [11] and Lin [6]. Naturally, a subject is to consider their unified extensions both in classical case and in nonclassical case. In this paper, we introduce some new concepts of generating spaces of quasi-norm family, and establish some new unified versions of Schauder-type fixed point theorems in more general setting. As applications, we also study the existence problems concerning the fixed points for operators on Menger probabilistic normed space and fuzzy normed space. Our results contain not only the former versions of the Schauder-type fixed point theorems but also the corresponding theorems in Menger probabilistic normed spaces and fuzzy normed spaces as their special cases.

\section{Fixed point theorems in generating spaces of quasi-norm family}

Throughout this paper we denote the set of all positive integers by $Z^{+}$and the field of real or complex numbers by $E$.

Hindawi Publishing Corporation

Fixed Point Theory and Applications

Volume 2006, Article ID 61623, Pages 1-10

DOI 10.1155/FPTA/2006/61623 
2 Fixed point theorems in generating spaces

Definition 2.1. Let $X$ be a linear space over $E$ and $\theta$ the origin of $X$. Let

$$
Q=\left\{|\cdot|_{\alpha}: \alpha \in(0,1]\right\}
$$

be a family of mappings from $X$ into $[0,+\infty) .(X, Q)$ is called a generating space of quasinorm family and $Q$ a quasi-norm family if the following conditions are satisfied:

(QN-1) $|x|_{\alpha}=0$ for all $\alpha \in(0,1]$ if and only if $x=\theta$;

$(\mathrm{QN}-2)|e x|_{\alpha}=|e||x|_{\alpha}$ for $x \in X$ and $e \in E$;

(QN-3) for any $\alpha \in(0,1]$ there exists a $\beta \in(0, \alpha]$ such that

$$
|x+y|_{\alpha} \leq|x|_{\beta}+|y|_{\beta} \quad \text { for } x, y \in X
$$

(QN-4) for any $x \in X,|x|_{\alpha}$ is non-increasing and left-continuous for $\alpha \in(0,1]$.

$(X, Q)$ is called a generating space of sub-strong quasi-norm family, strong quasi-norm family and semi-norm family respectively, if (QN-3) is strengthened to (QN-3u), (QN$3 \mathrm{t})$ and $(\mathrm{QN}-3 \mathrm{e})$, where

(QN-3u) for any $\alpha \in(0,1]$ there exists a $\beta \in(0, \alpha]$ such that

$$
\left|\sum_{i=1}^{n} x_{i}\right|_{\alpha} \leq \sum_{i=1}^{n}\left|x_{i}\right|_{\beta} \quad \text { for any } n \in Z^{+}, x_{i} \in X(i=1,2, \ldots, n) \text {; }
$$

(QN-3t) for any $\alpha \in(0,1]$ there exists a $\beta \in(0, \alpha]$ such that

$$
|x+y|_{\alpha} \leq|x|_{\alpha}+|y|_{\beta} \quad \text { for } x, y \in X
$$

(QN-3e) for any $\alpha \in(0,1]$, it holds that $|x+y|_{\alpha} \leq|x|_{\alpha}+|y|_{\alpha}$ for $x, y \in X$.

Remark 2.2. Clearly, by Definition 2.1 we obtain the following assertions: (QN-3e) implies (QN-3t); (QN-3t) and (QN-4) imply (QN-3u); (QN-3u) implies (QN-3).

Lemma 2.3. Let $(X, Q)$ be a generating space of quasi-norm family, $\varepsilon>0, \alpha \in(0,1], N(\varepsilon, \alpha)$ $=\left\{x:|x|_{\alpha}<\varepsilon\right\}$. Then

(i) $e \neq 0$ implies $e N(1, \alpha)=N(|e|, \alpha)$;

(ii) $\varepsilon_{1} \leq \varepsilon_{2}$ implies $N\left(\varepsilon_{1}, \alpha\right) \subset N\left(\varepsilon_{2}, \alpha\right)$;

(iii) $\alpha_{1} \leq \alpha_{2}$ implies $N\left(\varepsilon, \alpha_{1}\right) \subset N\left(\varepsilon, \alpha_{2}\right)$.

Proof. It follows immediately from (QN-2) and (QN-4).

Lemma 2.4. Let $(X, Q)$ be a generating space of quasi-norm family. Then there exists a topology $\mathscr{T}_{Q}$ on $X$ such that $\left(X, \mathscr{T}_{Q}\right)$ is a first-countable Hausdorff linear topological space (further, is metrizable) having $\{N(\varepsilon, \alpha): \varepsilon>0, \alpha \in(0,1]\}$ as a neighbourhood base of $\theta$. Additionally, if $(X, Q)$ is a generating space of semi-norm family, then $\left(X, \mathscr{T}_{Q}\right)$ is a locally convex space.

Sketch of proof. Applying Lemma 2.3, we have the following.

(a) For $N\left(\varepsilon_{1}, \alpha_{1}\right)$ and $N\left(\varepsilon_{2}, \alpha_{2}\right)$ there is a $N\left(\varepsilon_{0}, \alpha_{0}\right)$ such that

$$
N\left(\varepsilon_{0}, \alpha_{0}\right) \subset N\left(\varepsilon_{1}, \alpha_{1}\right) \cap N\left(\varepsilon_{2}, \alpha_{2}\right),
$$


where

$$
\varepsilon_{0}=\alpha_{0}=\min \left\{\varepsilon_{1}, \alpha_{1}, \varepsilon_{2}, \alpha_{2}\right\}
$$

(b) For $N(\varepsilon, \alpha)$, by $(\mathrm{QN}-3)$ and $(\mathrm{QN}-4)$, there is an $N(\varepsilon / 2, \beta)$ such that

$$
N(\varepsilon / 2, \beta)+N(\varepsilon / 2, \beta) \subset N(\varepsilon, \alpha) .
$$

(c) For any $e \in E,|e| \leq 1$, it holds that $e N(\varepsilon, \alpha) \subset N(\varepsilon, \alpha)$.

(d) For any $x \in X$, there is a $e=\varepsilon /\left(|x|_{\alpha}+\varepsilon\right) \in E$ such that $e x \in N(\varepsilon, \alpha)$.

(e) For $\theta \neq x \in X$, by (QN-1), there exist $\alpha_{0} \in(0,1]$ and $\varepsilon_{0}>0$ such that

$$
|x|_{\alpha_{0}}>\varepsilon_{0} \text {, that is, } x \notin N\left(\varepsilon_{0}, \alpha_{0}\right) \text {. }
$$

(f) $\left\{N(1 / n, 1 / n): n \in Z^{+}\right\}$is also a neighbourhood base of $\theta$ for $\mathscr{T}_{Q}$.

(g) If $(X, Q)$ satisfies $(\mathrm{QN}-3 \mathrm{e})$, then $N(\varepsilon, \alpha)$ is convex.

Finally, by [4, pages 34-35, pages 45-49], the assertion is valid.

Remark 2.5. From Lemma 2.4 we see that the topology $\mathscr{T}_{Q}$ can be described using sequence instead of net or filter.

Definition 2.6. Let $(X, Q)$ be a generating space of quasi-norm family.

(i) A sequence $\left\{x_{n}\right\}_{n=1}^{\infty} \subset X$ is said

(a) to converge to $x \in X$ denoted by $\lim _{n \rightarrow \infty} x_{n}=x$ if $\lim _{n \rightarrow \infty}\left|x_{n}-x\right|_{\alpha}=0$ for each $\alpha \in(0,1]$ (equivalently, for each $\alpha \in(0,1]$ there is a $K \in Z^{+}$such that $\mid x_{n}-$ $\left.x\right|_{\alpha}<\alpha$ for all $\left.n \geq K\right)$;

(b) to be a Cauchy sequence if $\lim _{m, n \rightarrow \infty}\left|x_{m}-x_{n}\right|_{\alpha}=0$ for each $\alpha \in(0,1]$.

(ii) A subset $B \subset X$ is said

(a) to be complete if every Cauchy sequence in $B$ converges in $B$;

(b) to be bounded if for each $\alpha \in(0,1]$ there is a $M=M(\alpha)>0$ such that $B \subset$ $N(M, \alpha)$;

(c) to be precompact (or totally bounded) if for each $\alpha \in(0,1]$ there exist $n_{\alpha} \in Z^{+}$ and $\left\{x_{1 \alpha}, x_{2 \alpha}, \ldots, x_{n_{\alpha} \alpha}\right\} \subset B$ such that $B \subset \bigcup_{i=1}^{n_{\alpha}} x_{i \alpha}+N(\alpha, \alpha)$;

(d) to be compact if every open cover of $B$ has a finite subcover.

(iii) An operator $T$ from $B \subset X$ into $X$ is said to be continuous if for each $x \in B$, $\lim _{n \rightarrow \infty} x_{n}=x$ implies $\lim _{n \rightarrow \infty} T x_{n}=T x$.

Remark 2.7. From Definition 2.6 and Lemma 2.4 we get the following immediately: if $B$ is compact, then it is precompact; If $B$ is precompact, then it is bounded; If $B$ is a subset of a precompact set, then it is also precompact.

LEMMA 2.8. Let $(X, Q)$ be a generating space of quasi-norm family.

(i) If $Y \subset X$ is a finite dimensional subspace of $X$, then $Y$ is topologically isomorphic to a finite dimensional Euclidean space and is therefore complete and closed in $X$.

(ii) A subset of $X$ is compact if and only if it is precompact and complete.

Proof. It follows from Lemma 2.4 and [4, pages 59-61]. 
4 Fixed point theorems in generating spaces

Lemma 2.9. Let $(X, Q)$ be a generating space of strong quasi-norm family. Then for each $\alpha \in(0,1],|x|_{\alpha}$ is a continuous function on $X$.

Proof. By (QN-3t), for $\left\{x_{n}\right\} \subset X$ and $x \in X$, we have

$$
|x|_{\alpha} \leq\left|x-x_{n}\right|_{\beta}+\left|x_{n}\right|_{\alpha}, \quad\left|x_{n}\right|_{\alpha} \leq\left|x_{n}-x\right|_{\beta}+|x|_{\alpha},
$$

that is, $\left.|| x_{n}\right|_{\alpha}-|x|_{\alpha}|\leq| x_{n}-\left.x\right|_{\beta}$, showing the assertion is true.

In the sequel, we denote the closure of a set $B$ by $\bar{B}$, the convex hull of $B$ by $\operatorname{co} B$ and the closure of the convex hull of $B$ by $\overline{c o} B$. Now, we give our main theorems.

THeORem 2.10. Let $(X, Q)$ be a generating space of sub-strong quasi-norm family satisfying that each $|\cdot|_{\alpha} \in Q$ is continuous on $X, C$ a compact convex subset of $X$ and $T$ a continuous operator from $C$ into $C$. Then there exists an $x_{0} \in C$ such that $T x_{0}=x_{0}$.

Proof. For $n \in Z^{+}$and $\alpha_{n} \in(0,1 / n]$, by $(\mathrm{QN}-3 \mathrm{u})$, there is $\beta_{n} \in\left(0, \alpha_{n}\right]$ such that

$$
\left|\sum_{i=1}^{k} x_{i}\right|_{\alpha_{n}} \leq \sum_{i=1}^{k}\left|x_{i}\right|_{\beta_{n}} ; \quad \forall k \in Z^{+}, \forall\left\{x_{i}\right\}_{i=1}^{k} \subset X .
$$

Set $\alpha_{n+1}=\min \left\{\beta_{n}, 1 /(n+1)\right\}$. By (QN-4), we have that $\left\{\alpha_{n}\right\}_{n=1}^{\infty} \subset(0,1]$ with $\alpha_{n+1} \leq \alpha_{n}$ and $\alpha_{n} \leq 1 / n$ such that

$$
\left|\sum_{i=1}^{k} x_{i}\right|_{\alpha_{n}} \leq \sum_{i=1}^{k}\left|x_{i}\right|_{\alpha_{n+1}} ; \quad \forall k \in Z^{+}, \forall\left\{x_{i}\right\}_{i=1}^{k} \subset X .
$$

Observe that a subset of a precompact set is also precompact. Since $C$ is compact and $T C \subset C$, there exist $p_{n} \in Z^{+}$and $\left\{y_{i n}\right\}_{i=1}^{p_{n}} \subset T C$ such that

$$
T C \subset \bigcup_{i=1}^{p_{n}} y_{i n}+N\left(\alpha_{n+1}, \alpha_{n+1}\right) .
$$

Set $g_{i n}(x)=\max \left\{0, \alpha_{n+1}-\left|T x-y_{i n}\right|_{\alpha_{n+1}}\right\}, \forall x \in C, i=1,2, \ldots, p_{n}$. Since the quasi-norms are continuous on $X$ and $T$ is continuous on $C$, we have that $g_{i n}(x)$ is continuous on $C$. If $x \in C$, then by (2.12), there exists $i_{0}\left(1 \leq i_{0} \leq p_{n}\right)$ such that $\left|T x-y_{i_{0} n}\right|_{\alpha_{n+1}}<\alpha_{n+1}$, that is, $g_{i_{0} n}(x)>0$. Set $g_{n}(x)=\sum_{i=1}^{p_{n}} g_{i n}(x)$. Then for all $x \in C, g_{n}(x)>0$. Define

$$
T_{n} x=\sum_{i=1}^{p_{n}} \frac{g_{\text {in }}(x)}{g_{n}(x)} y_{i n}, \quad x \in C .
$$

Then $T_{n}$ is a continuous operator on $C$. Notice that $g_{i n}(x) \neq 0$ if and only if $\left|T x-y_{i n}\right|_{\alpha_{n+1}}$ $<\alpha_{n+1}$. For each $x \in C$, by (2.11) and (QN-2) we have that

$$
\begin{aligned}
\left|T x-T_{n} x\right|_{\alpha_{n}} & =\left|\frac{1}{g_{n}(x)} \sum_{i=1}^{p_{n}} g_{i n}(x)\left(T x-y_{i n}\right)\right|_{\alpha_{n}} \\
& \leq \frac{1}{g_{n}(x)} \sum_{i=1}^{p_{n}} g_{i n}(x)\left|T x-y_{i n}\right|_{\alpha_{n+1}}<\alpha_{n+1} .
\end{aligned}
$$


Set $C_{n}=\overline{\mathrm{co}}\left\{y_{i n}\right\}_{i=1}^{p_{n}}, Y_{n}=\operatorname{span}\left\{y_{i n}\right\}_{i=1}^{p_{n}}$. Since $C$ is compact and convex, by (2.13) we have that $T_{n} C \subset C_{n}$ and $C_{n} \subset C$. Thus, $T_{n} C_{n} \subset C_{n}$. Since $Y_{n}$ is a finite dimensional closed subspace of $X$ and the bounded convex closed set $C_{n} \subset Y_{n}$, by the Brouwer fixed point theorem and Lemma 2.8(i), there exists $x_{n} \in C_{n}$ such that $T_{n} x_{n}=x_{n}$. Since $\left\{x_{n}\right\}_{n=1}^{\infty} \subset C$ and $C$ is compact, without loss of generality, we can suppose that $\lim _{n \rightarrow \infty} x_{n}=x_{0} \in C$. For each $\alpha \in(0,1]$, by $(\mathrm{QN}-3 \mathrm{u})$ and $(\mathrm{QN}-4)$, there exists $\beta \in(0, \alpha / 3]$ such that

$$
\left|x_{0}-T x_{0}\right|_{\alpha} \leq\left|x_{0}-x_{n}\right|_{\beta}+\left|T_{n} x_{n}-T x_{n}\right|_{\beta}+\left|T x_{n}-T x_{0}\right|_{\beta}
$$

Since $T$ is continuous and $\lim _{n \rightarrow \infty} \alpha_{n}=0$, there exists a $K \in Z^{+}$such that $\alpha_{n} \leq \beta, \mid x_{0}-$ $\left.x_{n}\right|_{\beta}<\beta$ and $\left|T x_{n}-T x_{0}\right|_{\beta}<\beta$ for all $n \geq K$. By (2.14) we have that

$$
\left|T_{n} x_{n}-T x_{n}\right|_{\beta} \leq\left|T_{n} x_{n}-T x_{n}\right|_{\alpha_{n}}<\alpha_{n+1} \leq \alpha_{n}<\beta
$$

Hence (2.15) implies $\left|x_{0}-T x_{0}\right|_{\alpha}<3 \beta \leq \alpha$, that is, $T x_{0}=x_{0}$. This completes the proof.

As a direct consequence of Theorem 2.10, we can obtain the following by Lemma 2.9.

Corollary 2.11. Let $(X, Q)$ be a generating space of strong quasi-norm family, $C$ a compact convex subset of $X$ and $T$ a continuous operator from $C$ into $C$. Then there exists an $x_{0} \in C$ such that $T x_{0}=x_{0}$.

THeOREM 2.12. Let $(X, Q)$ be a generating space of sub-strong quasi-norm family satisfying that each $|\cdot|_{\alpha} \in Q$ is continuous on $X$. Let $C$ be a closed convex subset of $X$ and $T$ a continuous operator from $C$ into $C$. If $X$ is complete and $\overline{T C}$ compact, then there exists an $x_{0} \in C$ such that $T x_{0}=x_{0}$.

Proof. Set $B=T C$. We will prove that $\overline{c o} B$ is compact. For each $\alpha \in(0,1]$, applying (QN$3 \mathrm{u})$ and $(\mathrm{QN}-4)$, there exists a $\beta \in(0, \alpha / 3)$ such that $\left|w_{1}+w_{2}+w_{3}\right|_{\alpha} \leq\left|w_{1}\right|_{\beta}+\left|w_{2}\right|_{\beta}+$ $\left|w_{3}\right|_{\beta}$ for all $w_{1}, w_{2}, w_{3} \in X$. Thus,

$$
N(\beta, \beta)+N(\beta, \beta)+N(\beta, \beta) \subset N(\alpha, \alpha)
$$

Applying (QN-3u) again, there exists a $\gamma \in(0, \beta]$ such that

$$
\left|\sum_{i=1}^{n} z_{i}\right|_{\beta} \leq \sum_{i=1}^{n}\left|z_{i}\right|_{\gamma} ; \quad \forall n \in Z^{+}, \forall\left\{z_{i}\right\}_{i=1}^{n} \subset X .
$$

Since $\overline{T C}$ is compact, we obtain that $B$ is precompact. Thus, there exist an $n_{\gamma} \in Z^{+}$and $\left\{x_{i \gamma}\right\}_{i=1}^{n_{y}} \subset B$ such that

$$
B \subset \bigcup_{i=1}^{n_{\gamma}} x_{i \gamma}+N(\gamma, \gamma)
$$


Suppose that $x \in \operatorname{co} B$. Then $x=\sum_{j=1}^{k} e_{j} y_{j}$, where $k \in Z^{+}, y_{j} \in B$ and $e_{j} \geq 0(j=1,2, \ldots, k)$, $\sum_{j=1}^{k} e_{j}=1$. By (2.19), there exists $x_{j} \in\left\{x_{i \gamma}\right\}_{i=1}^{n_{\gamma}}$ such that $y_{j}-x_{j} \in N(\gamma, \gamma),(j=1,2, \ldots, k)$. By (2.18), we have that

$$
\left|x-\sum_{j=1}^{k} e_{j} x_{j}\right|_{\beta} \leq \sum_{j=1}^{k} e_{j}\left|y_{j}-x_{j}\right|_{\gamma}<\gamma \leq \beta .
$$

Set $C_{\gamma}=\operatorname{co}\left\{x_{i \gamma}\right\}_{i=1}^{n_{\gamma}}, Y_{\gamma}=\operatorname{span}\left\{x_{i \gamma}\right\}_{i=1}^{n_{\gamma}}$. Since $\sum_{j=1}^{k} e_{j} x_{j} \in C_{\gamma}$, from (2.20) we get that

$$
x \in C_{\gamma}+N(\beta, \beta), \quad \text { that is, } \operatorname{co} B \subset C_{\gamma}+N(\beta, \beta) \text {. }
$$

Since $Y_{\gamma}$ is a finite dimensional space and $C_{\gamma}$ a bounded set of $Y_{\gamma}$, we derive that $\bar{C}_{\gamma}$ is compact. Thus, there exist $k_{\gamma} \in Z^{+}$and $\left\{z_{i \gamma}\right\}_{i=1}^{k_{\gamma}} \subset \bar{C}_{\gamma} \subset \overline{\mathrm{co}} B$ such that $C_{\gamma} \subset \bar{C}_{\gamma} \subset \bigcup_{i=1}^{k_{\gamma}} z_{i \gamma}+$ $N(\beta, \beta)$. Hence, by (2.17) and (2.21) we have that

$$
\begin{aligned}
\overline{\operatorname{co}} B & \subset \operatorname{co} B+N(\beta, \beta) \subset C_{\gamma}+N(\beta, \beta)+N(\beta, \beta) \\
& \subset \bigcup_{i=1}^{k_{y}} z_{i \gamma}+N(\beta, \beta)+N(\beta, \beta)+N(\beta, \beta) \subset \bigcup_{i=1}^{k_{y}} z_{i \gamma}+N(\alpha, \alpha),
\end{aligned}
$$

showing $\overline{\mathrm{co}} B$ is precompact. Since $X$ is complete, we obtain that $\overline{\mathrm{co}} B$ is complete. Applying Lemma 2.8(ii), $\overline{\mathrm{co}} B$ is compact. Since $B \subset C$ and $C$ is a closed convex set, we derive that $\overline{\mathrm{co}} B \subset C$. Clearly, $T(\overline{\mathrm{co}} B) \subset T C=B \subset \overline{\mathrm{co}} B$. Therefore, $T$ is a continuous operator from the convex compact set $\overline{\mathrm{co}} B$ into itself. Applying Theorem 2.10, there exists $x_{0} \in \overline{\mathrm{co}} B \subset C$ such that $T x_{0}=x_{0}$. This completes the proof.

As a direct consequence of Theorem 2.12, we can obtain the following by Lemma 2.9. Corollary 2.13. Let $(X, Q)$ be a generating space of strong quasi-norm family. Let $C$ be a closed convex subset of $X$ and $T$ a continuous operator from $C$ into $C$. If $X$ is complete and $\overline{T C}$ compact, then there exists an $x_{0} \in C$ such that $T x_{0}=x_{0}$.

TheOREM 2.14. Let $(X, Q)$ be a generating space of strong quasi-norm family and $C$ a closed convex subset of $X$. Let $T_{1}$ and $T_{2}$ be continuous operators from $C$ into $C$ satisfying the following conditions:

(i) $T_{1}$ is contractive, that is, there exists a constant $r \in[0,1)$ such that

$$
\left|T_{1} x-T_{1} y\right|_{\alpha} \leq r|x-y|_{\alpha} \quad \forall \alpha \in(0,1], x, y \in C \text {. }
$$

(ii) $\overline{T_{2} C}$ is compact.

(iii) For any $x, y \in C$ it holds that $T_{1} x+T_{2} y \in C$.

If $X$ is complete, then there exists an $x_{0} \in C$ such that $x_{0}=\left(T_{1}+T_{2}\right) x_{0}=T_{1} x_{0}+T_{2} x_{0}$.

Proof. Suppose that $z \in \overline{T_{2} C}$. Define

$$
T_{z}: T_{z} x=T_{1} x+z, \quad \forall x \in C
$$


Then $T_{z} x-T_{z} y=T_{1} x-T_{1} y$. Since $C$ is closed, by (i) and (iii) we have that $T_{z}$ is a contractive operator from $C$ into $C$. Set $y_{n+1}=T_{z} y_{n}$, where $n \in Z^{+}$and $y_{0} \in C$. Then $\left\{y_{n}\right\}_{n=0}^{\infty} \subset C$. Since (QN-3t) and (QN-4) imply (QN-3u), for each $\alpha \in(0,1]$, by (i) and $(\mathrm{QN}-3 \mathrm{u})$, there exists a $\beta \in(0, \alpha]$ such that

$$
\begin{aligned}
\left|y_{n+p}-y_{n}\right|_{\alpha} & =\left|\sum_{i=1}^{p}\left(y_{n+i}-y_{n+i-1}\right)\right|_{\alpha} \leq \sum_{i=1}^{p}\left|y_{n+i}-y_{n+i-1}\right|_{\beta} \\
& \leq \sum_{i=1}^{p} r^{n+i-1}\left|y_{1}-y_{0}\right|_{\beta} \leq \frac{r^{n}}{1-r}\left|y_{1}-y_{0}\right|_{\beta},
\end{aligned}
$$

showing that $\left\{y_{n}\right\}$ is a Cauchy sequence in $C$. Since $X$ is complete and $C$ closed, $\lim _{n \rightarrow \infty} y_{n}$ $=y \in C$. Observe that $T$ is continuous. From $y_{n+1}=T_{z} y_{n}$ we derive that $T_{z} y=y$. By (i) we see that $y$ is a unique fixed point of $T_{z}$. Define $S: S z=y$. Then $S$ is an operator from $\overline{T_{2} C}$ into $C$. By (2.24) we have that

$$
S z=T_{1} S z+z
$$

By (i) and (QN-3t), for each $\alpha \in(0,1]$ there is $\beta \in(0, \alpha]$ such that

$$
\begin{aligned}
\left|S z_{1}-S z_{2}\right|_{\alpha} & =\left|\left(T_{1} S z_{1}-T_{1} S z_{2}\right)+\left(z_{1}-z_{2}\right)\right|_{\alpha} \\
& \leq\left|\left(T_{1} S z_{1}-T_{1} S z_{2}\right)\right|_{\alpha}+\left|z_{1}-z_{2}\right|_{\beta} \leq r\left|S z_{1}-S z_{2}\right|_{\alpha}+\left|z_{1}-z_{2}\right|_{\beta}
\end{aligned}
$$

for all $z_{1}, z_{2} \in \overline{T_{2} C}$, that is, $\left|S z_{1}-S z_{2}\right|_{\alpha} \leq(1 /(1-r))\left|z_{1}-z_{2}\right|_{\beta}$, showing that $S$ is continuous. Since $S T_{2}$ is a continuous operator from $C$ into $C, S\left(\overline{T_{2} C}\right)$ is compact by (ii), and $\overline{S T_{2} C} \subset S\left(\overline{T_{2} C}\right)$, we derive that $\overline{S T_{2} C}$ is compact. Observe that (QN-3t) and (QN-4) imply $(\mathrm{QN}-3 \mathrm{u})$, and by Lemma 2.9, (QN-3t) implies the quasi-norms are continuous. Applying Theorem 2.12, there exists an $x_{0} \in C$ such that $S T_{2} x_{0}=x_{0}$. Setting $z_{0}=T_{2} x_{0}$, then $S z_{0}=x_{0}$, and by (2.26), $S z_{0}=T_{1} S z_{0}+z_{0}$. Therefore, $x_{0}=T_{1} x_{0}+T_{2} x_{0}$. This completes the proof.

Remark 2.15. From Lemmas 3.5 and 2.9 we see that, if $(X, Q)$ is a generating space of semi-norm family, then it is a locally convex Hausdorff linear topological space and its semi-norms are continuous. Noticing that (QN-3e) implies (QN-3u) and (QN-3t), Theorems 2.10, 2.12 (Corollaries 2.11, 2.13) and Theorem 2.14 are in some sense the generalizations of fixed point theorems (in locally convex space) of Schauder-Tychonoff, Schauder-Hukanare and Schauder-Krasnoselskii, respectively.

\section{Applications: fixed point theorems in probabilistic case and fuzzy case}

Throughout this section, We denote by $L, R, \Delta$ the mappings from $[0,1] \times[0,1]$ into $[0,1]$ which are symmetric and nondecreasing for both arguments and satisfy $L(0,0)=0$, $R(1,1)=1, \Delta(a, 1)=a$ and $\Delta(a, \Delta(b, c))=\Delta(\Delta(a, b), c)$, respectively. we denote by $\mathscr{F}$ the set of all fuzzy real numbers (see [9]). If $\eta \in \mathscr{F}$ and $\eta(t)=0$ for $t<0$, then $\eta$ is called a non-negative fuzzy real number and by $\mathscr{F}^{+}$we mean the set of all them. For $\eta \in \mathscr{F}^{+}$ and $\alpha \in(0,1], \alpha$-level set $[\eta]_{\alpha}=\{t: \eta(t) \geq \alpha\}$ is a closed interval and we write by $[\eta]_{\alpha}$ $=\left[\eta_{\alpha}^{-}, \eta_{\alpha}^{+}\right]$. We also denote by $\mathscr{D}$ the set of all left-continuous distribution functions (see 
$[7,10])$; by $\overline{0}$ the fuzzy number which satisfies $\overline{0}(t)=1$ for $t=0$ and $\overline{0}(t)=0$ for $t \neq 0$; by $H$ the distribution functions which satisfies $H(t)=1$ for $t>0$ and $H(t)=0$ for $t \leq 0$.

Now we recall some basic concepts and facts about fuzzy normed space (briefly, FNS) and probabilistic normed space (briefly, PNS).

Definition 3.1 (see $[2,9]$ ). Let $X$ be a real linear space and $\|\cdot\|$ a mapping from $X$ into $\mathscr{F}^{+}$. Denote $[\|x\|]_{\alpha}=\left[\|x\|_{\alpha}^{-},\|x\|_{\alpha}^{+}\right]$for $x \in X$ and $\alpha \in(0,1]$. The quadruple $(X,\|\cdot\|, L, R)$ is called an FNS and $\|\cdot\|$ a fuzzy norm if the following conditions are satisfied:

(FN-1) $\|x\|=\overline{0}$ if and only if $x=\theta$;

(FN-2) $\|e x\|=|e| \odot\|x\|$ for all $x \in X$ and $e \in(-\infty,+\infty)$;

(FN-3) for all $x, y \in X$,

(i) $\|x+y\|(s+t) \geq L(\|x\|(s),\|y\|(t))$ whenever $s \leq\|x\|_{1}^{-}, t \leq\|y\|_{1}^{-}$and $s+$ $t \leq\|x+y\|_{1}^{-}$

(ii) $\|x+y\|(s+t) \leq R(\|x\|(s),\|y\|(t))$ whenever $s \geq\|x\|_{1}^{-}, t \geq\|y\|_{1}^{-}$and $s+$ $t \geq\|x+y\|_{1}^{-}$.

Remark 3.2. By [9, Theorems 3.1-3.4] we know that the topology of $(X,\|\cdot\|, L, R)$ is decided by $\left\{\|x\|_{\alpha}^{+}: \alpha \in(0,1]\right\}$ or $\left\{N^{+}(\varepsilon, \alpha): \varepsilon>0, \alpha \in(0,1]\right\}$, where $N^{+}(\varepsilon, \alpha)=\left\{x:\|x\|_{\alpha}^{+}<\varepsilon\right\}$.

Lemma 3.3 (see [9]). Let $(X,\|\cdot\|, L, R)$ be an FNS. Suppose that

(R-1) $R \leq \max$;

(R-2) for each $\alpha \in(0,1]$ there exists a $\beta \in(0, \alpha]$ such that $R(\beta, \gamma)<\alpha$ for $\gamma \in(0, \alpha)$;

(R-3) $\lim _{a \rightarrow 0^{+}} R(a, a)=0$.

Then $\left(X,\left\{\|x\|_{\alpha}^{+}: \alpha \in(0,1]\right\}\right)$ is

(i) a generating space of quasi-norm family if $(X,\|\cdot\|, L, R)$ satisfies $(R-3)$;

(ii) a generating space of strong quasi-norm family if $(X,\|\cdot\|, L, R)$ satisfies $(R-2)$;

(iii) a generating space of semi-norm family if $(X,\|\cdot\|, L, R)$ satisfies $(R-1)$.

Definition 3.4 (see $[6,7,10]$ ). Let $X$ be a real linear space and $F$ a mapping from $X$ into D. Denote $F(x)(t)=f_{x}(t)$ for $x \in X$ and $t \in(-\infty,+\infty)$. The triple $(X, F, \Delta)$ is called a Menger PNS if the following conditions are satisfied:

$(\mathrm{PN}-1) f_{x}(0)=0 ; f_{x}(t)=H(t)$ if and only if $x=\theta$;

(PN-2) $f_{e x}(t)=f_{x}(t /|e|)$ for all $x \in X$ and $0 \neq e \in(-\infty,+\infty)$;

(PN-3) $f_{x+y}(s+t) \geq \Delta\left(f_{x}(s), f_{y}(t)\right)$ for all $x, y \in X$ and $s, t \geq 0$.

Lemma 3.5 (see $[8,10])$. Let $(X, F, \Delta)$ be a Menger PNS. For any $\varepsilon>0$ and $\alpha \in(0,1]$ we define $N^{*}(\varepsilon, \alpha)=\left\{x: f_{x}(\varepsilon)>1-\alpha\right\}$ and $\|x\|_{\alpha}=\inf \left\{t \geq 0: f_{x}(t)>1-\alpha\right\}$. Then

(i) $N^{*}(\varepsilon, \alpha)=\left\{x:\|x\|_{\alpha}<\varepsilon\right\}$;

(ii) $f_{x}(t) \geq f_{y}(t)$ for all $t \geq 0$ if and only if $\|x\|_{\alpha} \leq\|y\|_{\alpha}$ for all $\alpha \in(0,1]$.

Lemma 3.6 (see $[8,11])$. Let $(X, F, \Delta)$ be a Menger PNS. Suppose that

$(\Delta-1) \Delta=\min ;$

$(\Delta-2)$ for each $\alpha \in(0,1)$ there exists $a \beta \in[\alpha, 1)$ such that $\Delta(\beta, \gamma)>\alpha$ for $\gamma \in(\alpha, 1)$;

$(\Delta-3) \sup _{a<1} \Delta(a, a)=1$.

Then $\left(X,\left\{\|x\|_{\alpha}: \alpha \in(0,1]\right\}\right)$ is

(i) a generating space of quasi-norm family if $(X, F, \Delta)$ satisfies $(\Delta-3)$;

(ii) a generating space of strong quasi-norm family if $(X, F, \Delta)$ satisfies $(\Delta-2)$;

(iii) a generating space of semi-norm family if $(X, F, \Delta)$ satisfies $(\Delta-1)$. 
Remark 3.7. From Lemmas 3.5 and 3.3(i) we see that if $(X, F, \Delta)$ satisfies $(\Delta-3)$, then the $(\varepsilon, \alpha)$-topology on $(X, F, \Delta)$ induced by $\left\{N^{*}(\varepsilon, \alpha): \varepsilon>0, \alpha \in(0,1]\right\}$ coincides with the topology on $\left(X,\left\{\|x\|_{\alpha}: \alpha \in(0,1]\right\}\right)$.

Next we make use of Theorems 2.10, 2.12, 2.14 and Lemmas 3.3, 3.5, 3.6 to give some Schauder-type fixed point theorems in FNS and Menger PNS. The proofs are omitted here for the sake of brevity.

Theorem 3.8. Let $(X,\|\cdot\|, L, R)$ be an FNS with (R-2), C a compact convex subset of $X$ and $T$ a continuous operator from $C$ into $C$. Then there exists an $x_{0} \in C$ such that $T x_{0}=x_{0}$.

Theorem 3.9. Let $(X,\|\cdot\|, L, R)$ be an FNS with (R-2), $C$ a closed convex subset of $X$ and $T$ a continuous operator from $C$ into $C$. If $X$ is complete and $\overline{T C}$ compact, then there exists an $x_{0} \in C$ such that $T x_{0}=x_{0}$.

Theorem 3.10. Let $(X,\|\cdot\|, L, R)$ be an FNS with $(R-2)$, and $C$ a closed convex subset of $X$. Let $T_{1}$ and $T_{2}$ be continuous operators from $C$ into $C$ satisfying the following conditions:

(i) $T_{1}$ is contractive, that is, there exists a constant $r \in[0,1)$ such that

$$
\left\|T_{1} x-T_{1} y\right\|_{\alpha}^{+} \leq r\|x-y\|_{\alpha}^{+} \quad \forall \alpha \in(0,1], x, y \in X
$$

(ii) $\overline{T_{2} C}$ is compact.

(iii) For any $x, y \in C$ it holds that $T_{1} x+T_{2} y \in C$.

If $X$ is complete, then there exists an $x_{0} \in C$ such that $x_{0}=\left(T_{1}+T_{2}\right) x_{0}=T_{1} x_{0}+T_{2} x_{0}$.

Theorem 3.11. Let $(X, F, \Delta)$ be a Menger PNS with ( $\Delta-2), C$ a compact convex subset of $X$ and $T$ a continuous operator from $C$ into $C$. Then there exists an $x_{0} \in C$ such that $T x_{0}=x_{0}$. Theorem 3.12. Let $(X, F, \Delta)$ be a Menger PNS with ( $\Delta-2), C$ a closed convex subset of $X$ and $T$ a continuous operator from $C$ into $C$. If $X$ is complete and $\overline{T C}$ is compact, then there exists an $x_{0} \in C$ such that $T x_{0}=x_{0}$.

Theorem 3.13. Let $(X, F, \Delta)$ be a Menger PNS with ( $\Delta-2)$, and $C$ a closed convex subset of $X$. Let $T_{1}$ and $T_{2}$ be continuous operators from $C$ into $C$ satisfying the following conditions:

(i) $T_{1}$ is contractive, that is, there exists a constant $r \in(0,1)$ such that centerline $f_{T_{1} x-T_{1} y}(t) \geq f_{x-y}(t / r)$ for all $t \geq 0$

(ii) $\overline{T_{2} C}$ is compact;

(iii) for any $x, y \in C$, it holds that $T_{1} x+T_{2} y \in C$.

If $X$ is complete, then there exists an $x_{0} \in C$ such that $x_{0}=\left(T_{1}+T_{2}\right) x_{0}=T_{1} x_{0}+T_{2} x_{0}$.

Remark 3.14. Since $\Delta(a, a) \geq a$ for all $a \in[0,1]$ is equivalent to $\Delta=\min$, and $T$ is nonexpansive implies $T$ is continuous, Theorem 3.12 presents an improved version of [11, Theorem 3.2], moreover, Theorems 3.11 and 3.13 present a complementary version of [6, Theorems 2 and 4].

\section{Acknowledgment}

This project is supported by the Science Foundation of Nanjing University of Information Science and Technology. 
10 Fixed point theorems in generating spaces

\section{References}

[1] K. Fan, A generalization of Tychonoff's fixed point theorem, Mathematische Annalen 142 (1961), 305-310.

[2] J.-X. Fang and G.-A. Song, $\Phi$-contractor and the solutions for nonlinear operator equations in fuzzy normed spaces, Fuzzy Sets and Systems 121 (2001), no. 2, 267-273.

[3] V. I. Istrăţescu, Fixed Point Theory. An Introduction, Mathematics and Its Applications, vol. 7, D. Reidel, Dordrecht, 1981.

[4] J. L. Kelley and I. Namioka, Linear Topological Spaces, Graduate Texts in Mathematics, no. 36, Springer, New York, 1976.

[5] I. Krasnoselskii, et al., Approximation Solution of Operator Equations, Wolters-Noordhoff, Groningen, 1978.

[6] X. Lin, Fixed-point theorems in probabilistic normed linear spaces, Journal of Mathematics (Wuhan) 3 (1983), no. 1, 73-82 (Chinese).

[7] A. N. Serstnev, On the notion of a random normed space, Doklady Akademii Nauk USSR 149 (1963), no. 2, 280-283, Soviet Mathematics 4 (1963), no. 2, 388-391.

[8] J. Z. Xiao and X. H. Zhu, Probabilistic norm of operators and resonance theorems, Applied Mathematics and Mechanics 20 (1999), no. 7, 781-788.

[9] __ On linearly topological structure and property of fuzzy normed linear space, Fuzzy Sets and Systems 125 (2002), no. 2, 153-161.

[10] S. S. Zhang, Basic theory and applications of probabilistic metric spaces. I, Applied Mathematics and Mechanics 9 (1988), no. 2, 123-133.

[11] S. S. Zhang and J. L. Guo, Probability integrals, Gateaux differentials and the Schauder principle in probabilistic normed linear spaces, Journal of Sichuan University. Natural Science Edition 26 (1989), no. 2, 127-135 (Chinese).

Jian-Zhong Xiao: Department of Mathematics, Nanjing University of Information Science and Technology, Nanjing 210044, China

E-mail address: xiaojz@nuist.edu.cn

Xing-Hua Zhu: Department of Mathematics, Nanjing University of Information Science and Technology, Nanjing 210044, China

E-mail address: zhuxh@nuist.edu.cn 